\title{
Malignant Uveal Neoplasm
}

National Cancer Institute

\section{Source}

National Cancer Institute. Malignant Uveal Neoplasm. NCI Thesaurus. Code C6105.

A primary or metastatic malignant neoplasm that affects the choroid, ciliary body, or iris. 